\title{
Nonalcoholic Steatohepatitis in Bariatric Patients with a Diagnosis of Obstructive Sleep Apnea
}

\author{
Toby N. Weingarten ${ }^{a} \quad$ Carlos B. Mantilla ${ }^{a}$ James M. Swain ${ }^{b}$ \\ Michael L. Kendrick $^{b}$ Jeff M. Oberhansley ${ }^{a}$ Robert J. Burcham ${ }^{a}$ \\ Tarsila C.R. Ribeiro ${ }^{c, e}$ Kymberly D. Watt ${ }^{c}$ Darrell R. Schroeder ${ }^{d}$ \\ Bradly J. Narra Juraj Sprung ${ }^{\text {a }}$

\begin{abstract}
${ }^{a}$ Department of Anesthesiology, ${ }^{b}$ Department of Surgery, ${ }^{c}$ Division of Gastroenterology and Hepatology, ${ }^{d}$ Health Sciences Research, Division of Biostatistics, College of Medicine, Mayo Clinic, Rochester, MN, USA, ${ }^{e}$ Department of Medicine, Gastroenterology Center, University Hospital of the Federal University of Juiz de Fora, Juiz de Fora, Minas Gerais,
\end{abstract} \\ Brazil
}

\section{Key Words}

Fatty liver disease $\cdot$ Steatohepatitis $\cdot$ Metabolic syndrome

\begin{abstract}
Objective: To study a possible association between obstructive sleep apnea (OSA) severity, managed with noninvasive ventilation, and nonalcoholic steatohepatitis (NASH) in bariatric surgical patients. Methods: Medical records of 218 bariatric surgical patients who underwent liver biopsy were reviewed. OSA severity was determined from preoperative polysomnography (apnea-hypopnea index (AHI) $\leq 15$ no/mild OSA vs. AHI $\geq 16$ moderate/severe OSA). Patients diagnosed with OSA were prescribed noninvasive ventilation. Patients were categorized according to liver histopathology into 3 groups: i) no liver disease or simple steatosis, ii) mild NASH (steatosis with necroinflammation and mild fibrosis (stage 0-1)), and iii) advanced NASH (steatosis with necroinflammation and more advanced fibrosis (stage $\geq 2$ )). Results: 125 patients (57\%) had no/mild OSA, and 93 (43\%) had moderate/severe OSA. There was no difference in serum aminotransferases between patients by OSA severity classification. There was a high prevalence of hepatic histopathological abnormalities: $84 \%$ patients had steatosis, $57 \%$ had necroinflammation, $34 \%$ had fibrotic changes, and $14 \%$ had advanced NASH. There was no association between severity of NASH and severity of OSA. Conclusions: There is no association between stage of steatohepatitis and OSA severity among morbidly obese patients managed with noninvasive ventilation.




\section{Introduction}

Metabolic syndrome, a cluster of morbidities including obesity, hypertension, insulin resistance and hyperlipidemia, is a risk for the development of diabetes, atherosclerosis, and cardiovascular disease [1]. These conditions are prevalent among morbidly obese patients $[2,3]$. Metabolic syndrome has also been linked to increased risk for developing nonalcoholic fatty liver disease (NAFLD), a condition that encompasses a range of liver injuries from uncomplicated steatosis to severe nonalcoholic steatohepatitis (NASH). NASH histopathology is characterized with degrees of inflammation and accumulation of collagen (fibrosis) which can lead to cirrhosis [4-7]. It has been estimated that over 10-20 years one third of patients with early stages of NASH will progress to cirrhosis, and of these $25 \%$ will develop portal hypertension within 3 years [6].

NAFLD is present in about $80 \%$ of bariatric surgical patients [8]. In addition to a high prevalence of metabolic syndrome, approximately $80 \%$ of bariatric patients assessed with polysomnography (PSG) have obstructive sleep apnea (OSA) $[9,10]$. It has been hypothesized that nocturnal hypoxemia in patients with OSA may contribute to the development of NAFLD $[11,12]$. Thus, it is possible that in morbidly obese patients with metabolic syndrome, coexisting OSA may independently contribute to the pathogenesis of NAFLD [12,13]. Several studies examined the relationship between OSA and NAFLD, but findings are inconsistent. In one study [14], OSA was associated with increased serum aminotransferases levels, but not with NASH, but in another study [15] nocturnal oxyhemoglobin desaturation was a marker of latent steatohepatitis. Although the pathogenesis of NAFLD is not fully understood, a 'two-hit' theory was proposed [13]. A 'first-hit' is metabolic syndrome which alters fatty acid metabolism and insulin resistance leading to steatosis. The 'second-hit', which advances steatosis into steatohepatitis, is altered oxidative stress $[13,16]$. Intermittent hypoxia increases liver tissue peroxidation, hepatic myeloperoxidase, and inflammatory cytokines, which may increase lipogenesis and inhibit fat oxidation $[12,17,18]$. However, a recent report showed no association of NAFLD with OSA in bariatric patients in whom nocturnal desaturations were not treated preoperatively with continuous positive airway pressure (CPAP) [19]. Since morbidly obese patients have high prevalence of both OSA [10] and NAFLD [8] they are a suitable population for studying potential associations.

Bariatric surgery is a treatment of medically complicated obesity, and in preparation for surgery at our institution all patients undergo comprehensive evaluation. When indicated, their evaluation includes testing for OSA with overnight PSG [10]. In addition, liver biopsies are routinely performed during surgery. Accordingly, the purpose of this study was to examine the relationship between the severity of OSA and degree of histopathological liver changes.

\section{Patients and Methods}

Participants

Protocol approval was obtained from the Institutional Review Board at the Mayo Clinic, IRB Committee for Expedited Procedures; IRB\#: 10-002194, last approval date April 7, 2011. Consistent with Minnesota Statute 144.335 Subd. 3a. (d), we included only patients who have provided authorization for research use of their medical records. All indications for bariatric surgery were in accordance with the National Institutes of Health (NIH) Consensus Development Panel [20].

Study Protocol

Health records were reviewed of medically complicated obese patients who underwent bariatric surgery, had completed preoperative PSG testing, and had intraoperative liver biopsies performed between December 2003 and March 2009. To avoid inter-laboratory differences in PSG reporting, only patients who underwent testing at the Mayo Clinic Sleep Laboratory were included. 


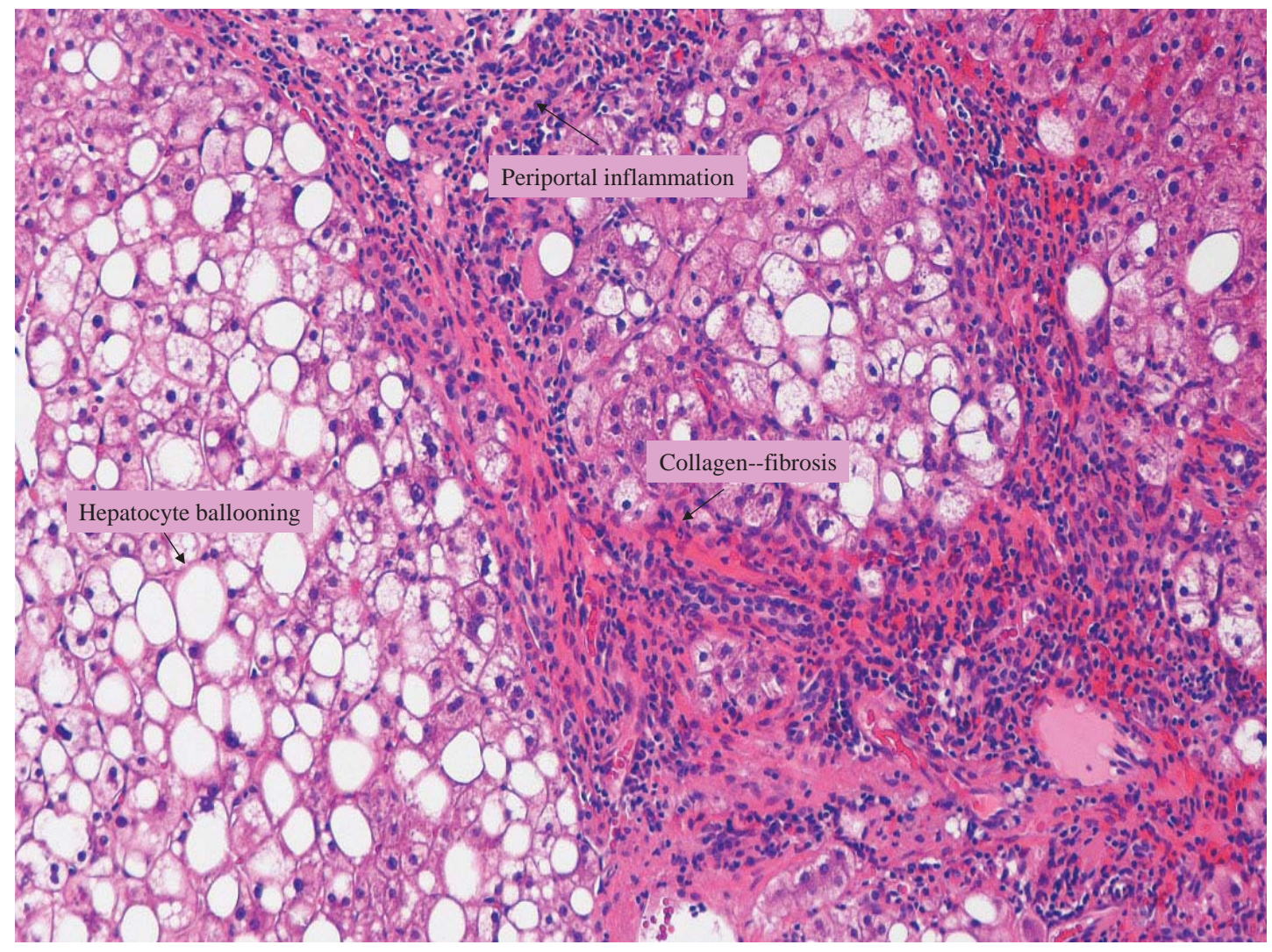

Fig. 1. Liver biopsy specimen (Masson's trichrome stain, $\times 100)$ showing macrovesicular steatosis, diffuse hepatocyte ballooning (HB) and rare Mallory's hyaline bodies (MB). Periportal inflammation and fibrosis suggest cirrhotic changes in liver parenchyma. These histopathological changes are consistent with advanced NASH.

\section{Quantification of NAFLD Severity}

Liver biopsy remains the 'gold standard' for establishing the definitive diagnosis of NAFLD/NASH, although changes in serum aminotransferases are useful for distinguishing NAFLD from other liver diseases [21]. Biopsy is routinely performed in patients undergoing bariatric surgery at our institution. Grading of liver injury was based on a previous separate study [8]. Patients were excluded if there was suspicion of liver disease other than NAFLD (i.e., history of alcohol abuse, viral hepatitis, use of hepatotoxic medications, liver storage diseases, etc.). Pathology reports from biopsy specimens were used for histopathological classification of NAFLD in the present study.

Liver biopsy evaluation included hematoxylin-eosin staining for assessment of parenchymal architecture, hepatocyte abnormalities, and inflammatory infiltrates [8]. The degree of fibrosis through collagen deposition was evaluated using Masson's trichome stain. Periodic-acid Schiff stain after diastase predigestion (PAS-D), reticulin, and iron stain were performed. Biopsy results were classified based on proposed systems for grading necroinflammatory activity (NIG) and fibrosis staging for NASH [22]. Significant histological liver lesions included steatosis, hepatocyte ballooning, intraacinar and portal inflammation, and fibrosis (fig. 1). Macrovesicular steatosis was graded 0 to 3 based on percent hepatocytes with steatosis $(0=$ none, grade $1=$ up to $33 \%$, grade $2=33-66 \%$ and grade $3=$ more than $66 \%)$. NIG for staging severity of steatohepatitis was 0 for no inflammation, and 1, 2 and 3 for mild, moderate and severe inflammation, respectively. To describe the location and extent of fibrosis, the following stages were used: stage 1 (fibrosis involving zone 3 perisinusoidal areas); stage 2 (perisinusoidal fibrosis with extension to periportal zone); stage 3 (with focal or bridging fibrosis), and stage 4 (cirrhosis). Patients were categorized into 3 groups: i) no NASH (normal liver histology or simple steatosis); ii) mild NASH (NASH with fibrosis grade $0-1$, and NIG $\geq 1$ ); and iii) advanced NASH (NASH with NIG and fibrosis stage $\geq 2$ ). 


\section{Quantification of OSA Severity}

Candidates for bariatric surgery undergo an assessment for the presence of OSA [10]. Evaluation is usually performed months before the operation. Patients with suspected OSA are referred for overnight oximetry and if warranted PSG, which are technologist-attended studies with data obtained via a computerized polygraph [10]. Electroencephalogram, electrooculogram, submental and anterior tibialis electromyogram, snoring by laryngeal microphone, oxyhemoglobin saturation $\left(\mathrm{SpO}_{2}\right)$, and respiratory effort were recorded. Airflow was analyzed by a nasal pressure transducer from December 2003 until August 2006 and after that time with both oronasal thermocouple and nasal pressure transducer. The PSG results were used to determine the apnea-hypopnea index (AHI). Hypopnea was defined by a $\geq 30 \%$ decrease in airflow for $\geq 10$ s despite respiratory effort and accompanied by $\geq 4 \%$ decrease in $\mathrm{SpO}_{2}$. Apnea was defined as $\geq 10$ s cessation of airflow. Patients found to have OSA underwent a second phase of PSG testing: a 'split study' where patients were fitted with a positive airway pressure device (typically CPAP). For analyses purposes severity of OSA was dichotomized according to the AHI using a modification of the American Academy of Sleep Medicine (AASM) criteria [23] as either: none or mild OSA (AHI $\leq 15$ ) versus moderate or severe OSA (AHI $\geq 16)$, consistent with several recent reports $[10,24,25]$. We also report mean $\mathrm{SpO}_{2}$, percent of total sleep time spent with $\mathrm{SpO}_{2}<90 \%$ for both initial and 'split' PSG testings. Patients diagnosed with OSA were prescribed CPAP and received regular follow-up in the Center for Sleep Medicine to encourage compliance.

\section{Data Collection}

From the electronic medical records demographic characteristics, comorbid conditions, preoperative variables, PSG results, preoperative liver transaminases, and histopathological findings of liver biopsies were abstracted using a standardized data collection form. All data were collected and managed using REDCap electronic data capture tools hosted at Mayo Clinic [26]. The following comorbid conditions were considered: hypertension (medically treated), coronary artery disease (medically treated or history of myocardial infarction, coronary stents/angioplasty, cardiac bypass surgery, or other interventions), history of congestive heart failure ( $\mathrm{EF}<40 \%$ or cor pulmonale), cerebrovascular disease (history of stroke or transient ischemic attacks), pulmonary disease (asthma, chronic obstructive or restrictive pulmonary disease, or pulmonary hypertension), and diabetes mellitus (treated with insulin or oral antihyperglycemic agents).

\section{Statistical Analysis}

Patient characteristics are presented overall and according to OSA category (none/mild vs. moderate/ severe). Continuous variables are summarized using mean \pm standard deviation (SD) or median and interquartile range (IQR). Categorical variables are presented as frequency percentages. These characteristics were compared across two OSA categories (none/mild vs. moderate/severe) using ANOVA (or Kruskal-Wallis test) for continuous variables and Fisher's exact test for categorical variables. Severity of NAFLD was compared across OSA groups using Fisher's exact test. The PSG test results were compared across NAFLD severity categories using Kruskal Wallis test. Statistical significance was established at two-tailed p values less than 0.05. Analyses were performed using SAS statistical software (Version 9.2, SAS Institute, Inc., Cary, NC, USA).

\section{Results}

Between December 2003 and March 2009, 218 adult patients underwent bariatric surgery, who were evaluated preoperatively by PSG and had intraoperative liver biopsy.

\section{Participants}

Patient demographic characteristics, comorbid conditions, and preoperative liver function tests are summarized in table 1 . Based on PSG results, 125 (57\%) patients had none/mild OSA, and 93 (43\%) patients had moderate/severe OSA. Patients with none/mild OSA were more likely to be females than those classified as having moderate/severe OSA $(p<0.05)$; otherwise, there were no other differences between the two groups. There were no differences in serum aspartate aminotransferase or alanine aminotransferase between OSA categories. 
Weingarten et al.: Nonalcoholic Steatohepatitis in Bariatric Patients with a Diagnosis of Obstructive Sleep Apnea

Table 1. Demographic and preoperative clinical characteristics of morbidly obese patients according to OSA categories

\begin{tabular}{|c|c|c|c|c|}
\hline \multirow[t]{2}{*}{ Demographics and preoperative characteristics } & \multirow[t]{2}{*}{ Total $(\mathrm{N}=218)$} & \multicolumn{2}{|l|}{ OSA* } & \multirow[t]{2}{*}{$\mathrm{p}$ value } \\
\hline & & $\begin{array}{l}\text { none/mild } \\
(\mathrm{N}=125)\end{array}$ & $\begin{array}{l}\text { moderate/severe } \\
(\mathrm{N}=93)\end{array}$ & \\
\hline Age, years & $48.2 \pm 10.8$ & $47.7 \pm 11.1$ & $48.9 \pm 10.4$ & 0.484 \\
\hline Female gender & $169(77.5)$ & $106(84.8)$ & $63(67.7)$ & 0.003 \\
\hline BMI, $\mathrm{kg} / \mathrm{m}^{2}$ & $47.9 \pm 9.1$ & $47.0 \pm 7.6$ & $49.2 \pm 10.8$ & 0.407 \\
\hline Superobese $\left(\mathrm{BMI} \geq 50 \mathrm{~kg} / \mathrm{m}^{2}\right)$ & $74(33.9)$ & $38(30.4)$ & $36(38.7)$ & 0.247 \\
\hline Diabetes mellitus & $73(33.5)$ & $39(31.2)$ & $34(36.6)$ & 0.469 \\
\hline Hypertension & $125(57.3)$ & $70(56)$ & $55(59.1)$ & 0.679 \\
\hline Coronary artery disease & $23(10.6)$ & $9(7.2)$ & $14(15.1)$ & 0.076 \\
\hline Heart failure & $5(2.3)$ & $4(3.2)$ & $1(1.1)$ & 0.396 \\
\hline Asthma & $52(23.9)$ & $29(23.2)$ & $23(24.7)$ & 0.873 \\
\hline COPD & $6(2.8)$ & $4(3.2)$ & $2(2.2)$ & 1.000 \\
\hline Cerebrovascular disease & $7(3.2)$ & $3(2.4)$ & $4(4.3)$ & 0.463 \\
\hline Serum creatinine & $0.9 \pm 0.3$ & $0.9 \pm 0.2$ & $0.9 \pm 0.3$ & 0.855 \\
\hline Serum AST, $\mathrm{U} / \mathrm{l}^{\dagger}$ & $28 \pm 12$ & $28 \pm 11$ & $28 \pm 13$ & 0.909 \\
\hline Patients with AST above normal & $16(8.3)$ & $9(8.0)$ & $7(8.8)$ & 1.000 \\
\hline Serum ALT, $U / \mathrm{l}^{\ddagger}$ & $37 \pm 22$ & $36 \pm 24$ & $38 \pm 19$ & 0.265 \\
\hline Patients with ALT above normal & $31(23.1)$ & $18(22.5)$ & $13(24.1)$ & 0.837 \\
\hline
\end{tabular}

${ }^{\text {aData }}$ are $\mathrm{N}(\%)$ or mean \pm standard deviation.

COPD = Chronic obstructive pulmonary disease; AST = aspartate aminotransferase; ALT = alanine aminotransferase

${ }^{\S}$ Characteristics were compared between groups using the rank sum test for continuous variables and Fisher's exact test for nominal variables.

${ }^{*}$ None/mild OSA (AHI $\left.\leq 15\right)$; moderate/severe OSA (AHI $\left.\geq 16\right)$.

${ }^{\dagger}$ Normal, 8-43 U/l; AST was available for 192 patients.

${ }^{\ddagger}$ Normal, 7-45 U/l; ALT was available for 134 patients.

\section{PSG Testing}

PSG test results are presented in table 2. From initial testing, as expected, those with moderate/severe OSA had worse results on PSG. Most patients ( $=185 ; 85 \%)$ had a split PSG test with the application of a noninvasive ventilation device. Regardless of OSA severity, following application of a CPAP, there was improvement in $\mathrm{AHI}$, mean $\mathrm{SpO}_{2}$ and percent of total sleep time spent with $\mathrm{SpO}_{2}<90 \%$. However, the differences between OSA groups remained statistically significant; those with moderate/severe OSA had more apneahypopnea (AHI) episodes per hour of sleep, lower mean $\mathrm{SpO}_{2}$, and spent more time with $\mathrm{SpO}_{2}$ $<90 \%$, compared to those with none/mild OSA. The use of a positive airway pressure device was deemed clinically useful in 177 (81.2\%) patients, and all were prescribed treatment with the CPAP / bi-level PAP device. Median time from PSG to surgery was 9.3 months (IQR 5-24 months). Of those patients prescribed CPAP before bariatric surgery, 140 (79.1\%) reported being compliant with use and 13 being noncompliant (9.3\%); compliance was not documented for 24 patients.

\section{Liver Biopsy Results}

From biopsy results, 94 (43.1\%) patients had normal liver histology or simple steatosis, 94 patients (43.1\%) had mild NASH, and $30(13.8 \%)$ had advanced NASH (fig. 1). There was no association between severity of NASH and severity of OSA (table 3). Similarly, there was no association between severity of NASH and PSG variables during the initial or 'split' PSG testing (table 4). In patients with moderate or severe OSA, an additional analysis was performed to 
Weingarten et al.: Nonalcoholic Steatohepatitis in Bariatric Patients with a Diagnosis of Obstructive Sleep Apnea

Table 2. Results of initial PSG testing and 'split test' in patients with none/mild and moderate/severe OSA ${ }^{\S}$

\begin{tabular}{|c|c|c|c|c|}
\hline \multirow[t]{2}{*}{ PSG testing } & \multirow[t]{2}{*}{ Total } & \multicolumn{2}{|l|}{ OSA* } & \multirow[t]{2}{*}{$\mathrm{p}$ value } \\
\hline & & none/mild & moderate/severe & \\
\hline Initial PSG testing & $(\mathrm{N}=218)$ & $(\mathrm{N}=125)$ & $(N=93)$ & \\
\hline AHI, number/h & $12(5,29)$ & $6(3,11)$ & $40(21,68)$ & $<0.001$ \\
\hline Mean $\mathrm{SpO}_{2}, \%$ & $93(92,94)$ & $94(92,95)$ & $92(90,94)$ & $<0.001$ \\
\hline Percent TST SpO $\mathrm{S}_{2}<90 \%$ & $2.9(0.4,10.3)$ & $1.1(0.1,3.6)$ & $9.8(3.0,32.3)$ & $<0.001$ \\
\hline Split PSG testing & $(\mathrm{N}=185)$ & $(\mathrm{N}=98)$ & $(\mathrm{N}=87)$ & \\
\hline AHI, number/h & $2(1,6)$ & $1(1,3)$ & $3(1,9)$ & $<0.001$ \\
\hline Mean $\mathrm{SpO}_{2}, \%$ & $94(93,96)$ & $95(93,96)$ & $94(92,95)$ & 0.003 \\
\hline Percent TST SpO $2<90 \%$ & $0.1(0.0,1.9)$ & $0.0(0.0,0.6)$ & $0.2(0.0,4.3)$ & 0.002 \\
\hline
\end{tabular}

TST = Total sleep time; $\mathrm{SpO}_{2}=$ oxyhemoglobin saturation measured by pulse oximetry.

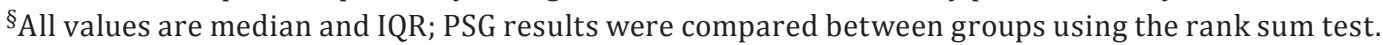
${ }^{*}$ None/mild OSA (AHI $\left.\leq 15\right)$; moderate/severe OSA (AHI $\geq 16$ ).

Table 3. Distribution of histopathological findings in patients with none/mild and moderate/severe OSA ${ }^{\S}$

\begin{tabular}{lllll}
\hline Liver histology category & Total $(\mathrm{N}=218)$ & OSA* & \multicolumn{2}{l}{$\mathrm{p}$ value } \\
\cline { 3 - 4 } & & $\begin{array}{l}\text { none/mild } \\
(\mathrm{N}=125)\end{array}$ & $\begin{array}{l}\text { moderate/severe } \\
(\mathrm{N}=93)\end{array}$ & \\
\hline No NASH & $94(43.1)$ & $55(44)$ & $39(41.9)$ & 0.435 \\
Mild NASH & $94(43.1)$ & $50(40)$ & $44(47.3)$ & $10(10.8)$ \\
Advanced NASH & $30(13.8)$ & $20(16)$ & 10 \\
\hline
\end{tabular}

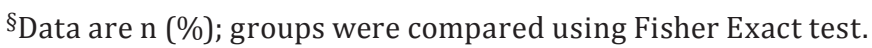

${ }^{*}$ None/mild OSA (AHI $\left.\leq 15\right)$; moderate/severe OSA (AHI $\left.\geq 16\right)$.

Table 4. PSG testing in patients with normal liver, mild NASH and advanced NASH: results of initial testing and 'split test'§

\begin{tabular}{|c|c|c|c|c|}
\hline PSG variable & No NASH & Mild NASH & Advanced NASH & $\mathrm{p}$ value ${ }^{*}$ \\
\hline Initial testing & $(\mathrm{N}=94)$ & $(\mathrm{N}=94)$ & $(\mathrm{N}=30)$ & \\
\hline $\mathrm{AHI}$, number/h & $13(5,28)$ & $12(6,29)$ & $8.5(4,43)$ & 0.551 \\
\hline Mean $\mathrm{SpO}_{2}, \%$ & $93(92,94)$ & $93.0(92,95)$ & $93.0(92,94)$ & 0.690 \\
\hline Percent TST SpO $\mathrm{S}_{2}<90 \%$ & $3.0(0.6,10.7)$ & $2.9(0.2,8.3)$ & $2.0(0.2,14.6)$ & 0.630 \\
\hline Split PSG & $(\mathrm{N}=76)$ & $(\mathrm{N}=86)$ & $(\mathrm{N}=24)$ & \\
\hline $\mathrm{AHI}$, number $/ \mathrm{h}$ & $2(1,5)$ & $2.0(1.0,6.0)$ & $2.5(1.0,6.5)$ & 0.377 \\
\hline Mean $\mathrm{SpO}_{2}, \%$ & $94(93,95)$ & $95(93,96)$ & $95(93,96)$ & 0.442 \\
\hline Percent TST $\mathrm{SpO}_{2}<90 \%$ & $0.2(0.0,2.7)$ & $0.1(0.0,0.5)$ & $0.6(0.1,3.9)$ & 0.061 \\
\hline
\end{tabular}

TST = Total sleep time; $\mathrm{SpO}_{2}=$ oxyhemoglobin saturation measured by pulse oximetry.

${ }^{\S}$ All values are median and IQR.

*Kruskal Wallis test. 
determine a possible association between CPAP / bi-level PAP treatment and NASH severity. 74 patients with moderate/severe OSA indicated to be compliant with use of a device for noninvasive ventilation. Of these, 30 (41\%) patients had normal liver histology and their median duration of treatment (i.e., time from PSG to surgery) was 15.4 (IQR 6.6-47.9) months. A further 37 (50\%) patients had mild NASH and were treated for a median (IQR) of 10.5 (5.850.7) months. Only 7 ( $9 \%$ ) patients had advanced NASH; they had received CPAP / bi-level PAP treatment for a median (IQR) of $10.6(6.9-42.8)$ months. No statistically significant difference in treatment duration was evident across NASH groups (Kruskal-Wallis $\mathrm{p}=0.856$ ).

\section{Discussion}

Our main finding is that among bariatric surgical patients preoperatively assessed to have OSA and prescribed CPAP when indicated, the severity of OSA was not associated with severity of liver histopathology (NASH severity). Rather, components of the metabolic syndrome appear to be primarily responsible for altered liver histopathology. While the use of CPAP can mitigate elevated serum aminotransferase levels in patients with NAFLD [27], it is unknown if CPAP can reverse or delay progression of 'metabolic' liver disease. It is unknown whether histopathology in our cohort would have been worse if patients with OSA had not used CPAP. However, there was no association between the duration of noninvasive ventilation treatment (CPAP or bi-level PAP device) and NASH severity in patients with moderate or severe OSA that reported being compliant with device use.

Morbidly obese patients have a high prevalence of both NAFLD and OSA [10, 12, 19]. In the present cohort, nearly $80 \%$ of patients had OSA, $84 \%$ had some degree of NAFLD, and $57 \%$ had NASH. The high coexistence of several risk factors for NAFLD may play significant role. For example, obesity is independently associated with insulin resistance and hypertriglyceridemia which contribute to development of steatosis [27-30]. Steatotic hepatocytes are sensitive to anoxia [31], and additional repetitive nocturnal oxyhemoglobin desaturation from OSA could accelerate hepatic injury [12].

There appears to be an association between elevated aminotransferases and sleeping disorders, but potential associations between the development of steatohepatitis and OSA have been inconsistent. Oxyhemoglobin desaturations are correlated with serum procollagen type III concentrations, a marker for latent fibrosis [15]. Severe untreated OSA has been found to be a risk for steatohepatitis and aminotransferases elevation, independent of body weight [11]. Jouet et al. [14] reported a positive association between OSA and increased aminotransferases in morbidly obese patients, but no association with NASH. Norman et al. [32] reported that serum aminotransferase levels were better predicted by oxyhemoglobin desaturation than by components of the metabolic syndrome. In that study, nadir $\mathrm{SpO}_{2}$ was more predictive than mean $\mathrm{SpO}_{2}$, suggesting that severity of hypoxemia was more important than sustained levels of moderate hypoxemia [32]. Chin et al. [27] demonstrated that the use of oxygen via noninvasive ventilation in obese patients promptly decreased elevations of aminotransferases. However, a prospective randomized trial in patients with OSA found that both the use of CPAP or sham CPAP reduced aminotransferase levels, which was attributed to 'changes in lifestyle after entering in the study', but behavioral changes were not specifically measured [33]. In our patients aminotransferases generally ranged from normal to slightly elevated, and there were no differences between the two OSA groups. This suggests that severe oxyhemoglobin desaturations were attenuated in the entire cohort, presumably by CPAP. Unfortunately, serum aminotransferases were obtained independent of the timing of the sleep study; thus we are not able to determine whether CPAP use influenced aminotransferases. 
Kallwitz et al. [24] reported only a 'trend' for an association between OSA and liver inflammation and fibrosis, but did not report preoperative CPAP use. Mishra et al. [34] found that nadir $\mathrm{SpO}_{2}$ was independently associated with NASH, while AHI was not. Patients with NASH had higher CPAP settings, suggesting that subjects with NASH had more sleep-related hypoxemia; however, CPAP settings did not significantly differ with increasing severity of NASH (presence of fibrosis) [34]. Absence of association between OSA and NASH in our study reiterates findings by others $[14,19]$, who also did not find an association, even in untreated patients.

Our findings suggest that components of the metabolic syndrome play a dominant role in the development of hepatic injury in obese patients. However, it is challenging to differentiate potential effects of OSA (intermittent hypoxemia) or metabolic syndrome on the development of liver disease in the morbidly obese because of the high prevalence of both. Some of the major clinical features associated with severe NASH are higher BMI, increased insulin levels, higher insulin resistance, diabetes, and hypertension [35]. Therefore, the high coexistence of these comorbidities and OSA in these patients creates collinear conditions that may obscure potential relationships.

Our study has several strengths over previous studies. The sample size is the largest to date, and the study cohort was treated within a few years (December 2003 to March 2009). A very complete histopathological evaluation of liver specimens was conducted using up-todate classification criteria. We were adequately powered to find a pathological change. In addition, we included only patients who underwent PSG evaluation at a single sleep laboratory, ensuring uniform quantification.

On the other hand, this study has several important limitations. First, this is a retrospective study, and thus we cannot ascertain whether OSA treatment was effective at reducing episodes of oxyhemoglobin desaturation or modified markers of liver disease. The results of home CPAP use may have differed from those achieved in the 'split' PSG study. Since metabolic syndrome appears to be a major contributor to liver injury, we cannot differentiate whether absence of association between OSA and NASH was because of compliant CPAP use or lack of any association, as was suggested in a study of untreated patients [19]. Patients in our study were selected based on suspicion for the presence of OSA and subsequently underwent PSG study, introducing a selection bias. Therefore, our conclusions cannot be applied to patients with unrecognized or untreated OSA. Lastly, our results may not be generalized to NAFLD in patients who do not have medically complicated obesity.

In summary, in morbidly obese patients evaluated before bariatric surgery with PSG who were prescribed noninvasive ventilation, severity of OSA was not associated with NASH severity. Our findings suggest that NASH histopathology in this population primarily reflects specific risk factors associated with the metabolic syndrome, and not the OSA severity.

\section{Acknowledgment}

We are thankful to Dr. Thomas Smyrk, Associate Professor of Pathology, for sharing histopathology slides and advice and Miss Ashley Nadeau, (statistical programmer/analyst) for data management and statistical assistance.

\section{Funding}

This project was supported by the Department of Anesthesiology, College of Medicine, Mayo Clinic, Rochester, MN, 55905 and NIH/NCRR CTSA Grant Number UL1 RR024150. Its contents are solely the responsibility of the authors and do not necessarily represent the official views of the NIH. 
Weingarten et al.: Nonalcoholic Steatohepatitis in Bariatric Patients with a Diagnosis of Obstructive Sleep Apnea

Author Contribution: Toby N. Weingarten, M.D., Carlos B. Mantilla, M.D. Ph.D., Bradly J. Narr, M.D., Juraj Sprung, M.D., Ph.D., Kymberly D. Watt, M.D (study design, data analysis, writing of manuscript); James M. Swain, M.D., Michael L. Kendrick, M.D. (study design, writing of manuscript); Jeff M. Oberhansley, R.N., Robert J. Burcham, R.N. (planning the study design, data collection and data summary), Tarsila C.R. Ribeiro, M.D. (analysis of liver pathology and assigning the NASH classification); Darrell R. Schroeder, M.S.(planning the study, data analysis, interpretation of statistical findings, drafting of the results section).

\section{Diclosure Statement}

The authors declare that they have no conflict of interest.

\section{References}

1 Mihic M, Modi P: Metabolic syndrome - risk factors for atherosclerosis and diabetes. Curr Diabetes Rev 2008;4:122-128.

2 Sugerman HJ, Wolfe LG, Sica DA, et al: Diabetes and hypertension in severe obesity and effects of gastric bypass-induced weight loss. Ann Surg 2003;237:751-756.

3 Sjostrom L, Lindroos AK, Peltonen M, et al: Lifestyle, diabetes, and cardiovascular risk factors 10 years after bariatric surgery. N Engl J Med 2004;351:2683-2693.

- 4 Tiniakos DG, Vos MB, Brunt EM: Nonalcoholic fatty liver disease: pathology and pathogenesis. Annu Rev Pathol 2010;5:145-171.

- 5 Angulo P: Nonalcoholic fatty liver disease. N Engl J Med 2002;346:1221-1231.

6 Caldwell S, Argo C: The natural history of non-alcoholic fatty liver disease. Dig Dis 2010;28:162-168.

- 7 Clark JM, Diehl AM: Nonalcoholic fatty liver disease: an underrecognized cause of cryptogenic cirrhosis. JAMA 2003;289:3000-3004.

- 8 Ribeireiro T, Swain J, Sarr M, et al: NAFLD and insulin resistance do not increase the risk of postoperative complications among patients undergoing bariatric surgery - a prospective analysis. Obes Surg 2011;21: 310-315.

9 O’Keeffe T, Patterson EJ: Evidence supporting routine polysomnography before bariatric surgery. Obes Surg 2004;14:23-26.

10 Weingarten TN, Flores AS, McKenzie JA, et al: Obstructive sleep apnoea and perioperative complications in bariatric patients. Br J Anaesth 2011;106:131-139.

$\checkmark 11$ Tanne F, Gagnadoux F, Chazouilleres O, et al: Chronic liver injury during obstructive sleep apnea. Hepatology 2005;41:1290-1296.

$\checkmark 12$ Ahmed MH, Byrne CD: Obstructive sleep apnea syndrome and fatty liver: association or causal link? World J Gastroenterol 2010;16:4243-4252.

13 Day CP, James OF: Steatohepatitis: a tale of two ‘hits'? Gastroenterology 1998;114:842-845.

14 Jouet P, Sabate JM, Maillard D, et al: Relationship between obstructive sleep apnea and liver abnormalities in morbidly obese patients: a prospective study. Obes Surg 2007;17:478-485.

15 Tatsumi K, Saibara T: Effects of obstructive sleep apnea syndrome on hepatic steatosis and nonalcoholic steatohepatitis. Hepatol Res 2005;33:100-104.

-16 Vgontzas AN, Papanicolaou DA, Bixler EO, et al: Sleep apnea and day time sleepiness and fatigue: relation to visceral obesity, insulin resistance, and hypercytokinemia. J Clin Endocrinol Metab 2000;85:1151-1158.

-17 Savransky V, Bevans S, Nanayakkara A, et al: Chronic intermittent hypoxia causes hepatitis in a mouse model of diet-induced fatty liver. Am J Physiol Gastrointest Liver Physiol 2007;293:G871-877.

-18 Savransky V, Nanayakkara A, Vivero A, et al: Chronic intermittent hypoxia predisposes to liver injury. Hepatology 2007;45:1007-1013.

19 Daltro C, Cotrim HP, Alves E, et al: Nonalcoholic fatty liver disease associated with obstructive sleep apnea: just a coincidence? Obes Surg 2010;20:1536-1543.

20 NIH Conference. Gastrointestinal surgery for severe obesity. Consensus Development Conference Panel. Ann Intern Med 1991;115:956-961.

21 Angulo P: Nonalcoholic fatty liver disease; in Hauser SC, Pardi DS, Poterucha JJ (eds): Mayo Clinic Gastroenterology and Hepatology Broad Review. Rochester, Mayo Clinic Scientific Press, 2008, pp 407-418.

-22 Brunt EM, Janney CG, Di Bisceglie AM, et al: Nonalcoholic steatohepatitis: a proposal for grading and staging the histological lesions. Am J Gastroenterol 1999;94:2467-2474.

-23 Sleep-related breathing disorders in adults: recommendations for syndrome definition and measurement techniques in clinical research. The Report of an American Academy of Sleep Medicine Task Force. Sleep 1999;22:667-689. 
24 Kallwitz ER, Herdegen J, Madura J, et al: Liver enzymes and histology in obese patients with obstructive sleep apnea. J Clin Gastroenterol 2007;41:918-921.

25 Polotsky VY, Patil SP, Savransky V, et al: Obstructive sleep apnea, insulin resistance, and steatohepatitis in severe obesity. Am J Respir Crit Care Med 2009;179:228-234.

-26 Harris PA, Taylor R, Thielke R, et al: Research electronic data capture (REDCap) - a metadata-driven methodology and workflow process for providing translational research informatics support. J Biomed Inform 2009;42:377-381.

27 Chin K, Nakamura T, Takahashi K, et al: Effects of obstructive sleep apnea syndrome on serum aminotransferase levels in obese patients. Am J Med 2003;114:370-376.

28 Farrell GC, Larter CZ: Nonalcoholic fatty liver disease: from steatosis to cirrhosis. Hepatology 2006; 43:S99-S112.

29 Ip MS, Lam B, Ng MM, et al: Obstructive sleep apnea is independently associated with insulin resistance. Am J Respir Crit Care Med 2002;165:670-676.

-30 Punjabi NM, Sorkin JD, Katzel LI, et al: Sleep-disordered breathing and insulin resistance in middle-aged and overweight men. Am J Respir Crit Care Med 2002;165:677-682.

-31 Caraceni P, Ryu HS, Subbotin V, et al: Rat hepatocytes isolated from alcohol-induced fatty liver have an increased sensitivity to anoxic injury. Hepatology 1997;25:943-949.

-32 Norman D, Bardwell WA, Arosemena F, et al: Serum aminotransferase levels are associated with markers of hypoxia in patients with obstructive sleep apnea. Sleep 2008;31:121-126.

33 Kohler M, Pepperell JC, Davies RJ, et al: Continuous positive airway pressure and liver enzymes in obstructive sleep apnoea: data from a randomized controlled trial. Respiration 2009;78:141-146.

-34 Mishra P, Nugent C, Afendy A, et al: Apnoeic-hypopnoeic episodes during obstructive sleep apnoea are associated with histological nonalcoholic steatohepatitis. Liver Int 2008;28:1080-1086.

-35 Brunt EM, Kleiner DE, Wilson LA, et al: Portal chronic inflammation in nonalcoholic fatty liver disease (NAFLD): a histologic marker of advanced NAFLD-clinicopathologic correlations from the Nonalcoholic Steatohepatitis Clinical Research Network. Hepatology 2009;49:809-820. 